\title{
Compensatory proliferation and apoptosis-induced proliferation: a need for clarification
}

\author{
Cell Death and Differentiation (2013) 20, 181; doi:10.1038/cdd.2012.82; published online 22 June 2012
}

\section{Dear Editor,}

During the EMBO Programmed Cell Death in Model Organisms meeting that was held in Israel (February 2012), the topic of compensatory proliferation (CP) took an important place in conference presentations and animated discussions. Several scientists felt the need for a term clarification at a time when the importance of the findings made in Drosophila have greatly impacted the understanding of tumor repopulation during cancer irradiation and also the process of regeneration in vertebrates. The scientists agreed that the terms of CP have been used sometimes inappropriately to describe the process by which apoptotic cells mediate mitogenic signaling thus promoting proliferation of the surrounding cells.

The phenomenon of CP was originally described when studying the response of the wing imaginal disc of Drosophila to massive cell loss after irradiation (Supplementary Figure S1A). Yet, irradiated discs recovered to form adult structures of normal size and shape. It was clear that there had been additional proliferation to compensate for the cell loss.

More recently, several labs found that cells stimulated to undergo apoptosis were able to promote proliferation of neighboring cells. ${ }^{1-4}$ This suggested that apoptotic cells can release mitogenic signals to promote tissue repair and regeneration (Supplementary Figure S1B). Candidates for mitogens produced by apoptotic cells included the products of the signaling genes wingless $(w g)$ and decapentaplegic (dpp), which are activated under these conditions. ${ }^{4,5}$ However, it is important to mention that in those experiments the authors used 'undead' cells: in these, apoptosis is induced by stress treatments or by ectopic induction of pro-apoptotic genes, but the cells are kept alive by the caspase inhibitor P35. Under these conditions, there is no 'compensation' for cell loss because the cells do not die, and hence it is problematic to refer to this paradigm as 'compensatory proliferation'. On the other hand, the persistent secretion of the Dpp and Wg mitogens results in excessive cell proliferation and the generation of hyperplastic overgrowths in the discs. ${ }^{2,4,5}$

One important finding was that the secretion of $\mathrm{Wg}$ and $\mathrm{Dpp}$ is not limited to undead cells, but also occurs in 'genuine' apoptotic cells. ${ }^{1,3,4,6}$ These observations suggested that the proliferation mediated by apoptotic cells might have an important role in $\mathrm{CP}, 1,3,4$ as it would be instrumental in the restoration of normal size after massive cell death. However, flies carrying mutations in both $w g$ and $d p p$ are still capable of $\mathrm{CP}$ after high levels of $\mathrm{X}$-rays induced apoptosis. ${ }^{5}$ Therefore either additional signals from apoptotic cells or more complex mechanisms must contribute to CP. In contrast, the hyperplastic overgrowths triggered by 'undead' cells are dependent on $w g$ and $d p p$, as cells mutant for either wg or dpp cannot produce them.

The ability of apoptotic cells to secrete mitogenic signals may be of major significance in regeneration processes and in the development of tumors. The formation of a new head of Hydra after amputation requires Wnt3-mediated induction of proliferation by apoptotic cells close to the cut site. ${ }^{7}$ More recently, it was shown that PGE2 emanating from apoptotic cells stimulates the growth of surviving tumor cells after irradiation and thereby promotes the repopulation of the tumor. ${ }^{8}$ Thus, the understanding of apoptosis-induced proliferation may open a new avenue to improve cancer radiotherapy.

In conclusion, we recommend a precise and distinct use of the terms of $\mathrm{CP}$ and apoptosis-induced proliferation or mitogenic signaling. CP is the process by which an injured tissue recovers its original size and in which the role of apoptosis remains to be elucidated. Apoptosis-induced proliferation is a cellular process by which apoptotic cells secrete mitogens, which in turn promote proliferation of surrounding living cells.

\section{Conflict of Interest}

The authors declare no conflict of interest.

\section{B Mollereau ${ }^{\star 1}$, A Perez-Garijo ${ }^{2}$, A Bergmann ${ }^{3}$, M Miura $^{4}$, O Gerlitz ${ }^{5}, H D$ Ryoo $^{6}$, H Steller $^{\star, 2}$ and G Morata ${ }^{*, 7}$}

${ }^{1}$ Group Apoptosis and Neurogenetics, Laboratory of Molecular Biology of the Cell, CNRS UMR5239, Ecole Normale Supérieure de Lyon, UMS3444 Biosciences Lyon Gerland, Université de Lyon, Lyon 69007, France;

${ }^{2}$ Howard Hughes Medical Institute, Laboratory of Apoptosis and Cancer Biology, The Rockefeller University, New York, NY, USA;

${ }^{3}$ Department of Cancer Biology, University of Massachusetts Medical School, Worcester, MA, USA;

${ }^{4}$ Department of Genetics, Graduate School of Pharmaceutical Sciences, University of Tokyo, CREST, JST 7-3-1 Hongo, Bunkyo-ku, Tokyo 113-0033, Japan;

${ }^{5}$ Department of Developmental Biology and Cancer Research, Institute for Medical Research Israel-Canada, Hebrew University-Hadassah Medical School, Jerusalem 91120, Israel;

${ }^{6}$ Department of Cell Biology, New York University School of Medicine, New York, NY, USA and

${ }^{7}$ Centro de Biología Molecular, CSIC-UAM, Madrid, Spain

* Corresponding authors: B Mollereau, Group Apoptosis and Neurogenetics, Laboratory of Molecular Biology of the Cell, CNRS UMR5239, Ecole Normale Supérieure de Lyon, UMS3444 Biosciences Lyon Gerland, Université de Lyon, Lyon 69007, France. Tel: +33 47272 8163; Fax: +33 47272 8674; E-mail: bertrand.mollereau@ens-lyon.fr (or) G Morata, Centro de Biología Molecular CSIC-UAM, Universidad Autónoma de Madrid, 28049, Madrid, Spain. Tel: +34 91 1964696;

Fax: +34 91 1964420; E-mail: gmorata@ cbm.uam.es

(or) H Steller, Howard Hughes Medical Institute, Laboratory of Apoptosis and Cancer Biology, The Rockefeller University, New York, 10021 NY, USA.

Tel: +1 212327 7075; Fax: +1 212327 7076;

E-mail: steller@mail.rockefeller.edu.

1. Fan Y, Bergmann A. Dev Cell 2008; 14: 399-410

2. Kondo $S$ et al. Mol Cell Biol 2006; 26: 7258-7268

3. Perez-Garijo A, Martin FA, Morata G. Development 2004; 131: 5591-5598.

4. Ryoo HD, Gorenc T, Steller H. Dev Cell 2004; 7: 491-501.

5. Perez-Garijo A, Shlevkov E, Morata G. Development 2009; 136: 1169-1177.

6. Smith-Bolton RK et al. Dev Cell 2009; 16: 797-809.

7. Chera S et al. Dev Cell 2009; 17: 279-289.

8. Huang $Q$ et al. Nat Med 2011; 17: 860-866. 